\title{
Tagungsbericht
}

Valerie Claessen* und Adrian Schmidt

\section{Design und Evaluation von Informationssystemen}

\author{
15th International Symposium of Information Science (ISI 2017) Berlin, Germany, 13th-15th \\ March 2017
}

https://doi.org/10.1515/iwp-2017-0051

Das International Symposium of Information Science wird in einem zweijährlichen Turnus von verschiedenen Gastgebern ausgerichtet. 2017 fand die mittlerweile 15. ISI unter dem Motto „Everything Changes, Everything Stays the Same? Understanding Information Spaces“ an der Humboldt-Universität zu Berlin statt. ${ }^{1}$ Das dreitägige Programm bestand aus verschiedenen Keynotes, Paper Sessions und Panels, außerdem wurde am Abend des zweiten Tages der Gerhard-Lustig-Preis verliehen. Aus dieser übergroßen Fülle stellen die Autoren exemplarisch einzelne Präsentationen aus den Sessions „Designing Scholarly Information Systems“, „User Perceptions of Information Systems“ und „Virtual Assistants: A Study on the Usability and User Perception of Customer Service System for E-Commerce" vor.

Dabei werden die ausgewählten Berichte, die zugrundeliegenden Problemstellungen, die Methoden und die aus den Forschungsarbeiten resultierenden Ergebnisse kurz zusammengefasst. Dazu wird die Forschungsarbeit der beiden Autoren, die im Rahmen der dritten Session präsentiert wurde, angesprochen.

Die Session „Designing Scholarly Information Systems“ eröffneten Corinna Breitinger und Prof. Bela Gipp von der Universität Konstanz. Sie stellten in ihrem Paper „VirtualPatent - Enabling the Traceability of Ideas Shared Online Using Decentralized Trusted Timestamping” das sogenannte VirtualPatent vor, das Online-Inhalt jeglicher

1 M. Gäde, V. Trkulja \& V. Petras (Eds.), Everything Changes, Everything Stays the Same? Understanding Information Spaces. Proceedings of the 15th International Symposium of Information Science (ISI 2017). Glückstadt: Werner Hülsbusch.

*Kontaktperson: Valerie Claessen, Heinrich-Heine Universität Düsseldorf, Institut für Sprache und Information, Abteilung Informationswissenschaft, Universitätsstr. 1, 40225 Düsseldorf, E-Mail: valerie.claessen@uni-duesseldorf.de

Adrian Schmidt, Heinrich-Heine Universität Düsseldorf, Institut für Sprache und Information, Abteilung Informationswissenschaft, Universitätsstr. 1, 40225 Düsseldorf,

E-Mail:adrian.schmidt@uni-duesseldorf.de
Art dauerhaft auf einen ursprünglichen Autor zurückführt. Die bisher unzureichenden Möglichkeiten, online veröffentlichte und geteilte Inhalte zu schützen, hemmte Wissenschaftler darin, Informationen und wissenschaftliche Aussagen zu teilen (besonders auf Social Media Plattformen). Es bestand keine Möglichkeit, einen Post dauerhaft und sicher dem ursprünglichen Autor zuzuordnen. Selbst wenn ein Zeitstempel gesetzt wurde, bestand das Risiko, dass dieser mit dem Verschwinden einer Plattform erlosch, oder der Post unerlaubt weiterverteilt wurde. Diese beiden essentiellen Bedingungen (Fälschungssicherheit und Beständigkeit) werden durch die neu entwickelte Methode des VirtualPatent sichergestellt. Das VirtualPatent versieht jeden von Nutzern geteilten Post mit einem Zeitstempel, der durch die Zuordnung eines Eintrages in der Blockchain der Kryptowährung Bitcoin vor Manipulationen geschützt wird. So wird das ursprüngliche Gedankengut einem Autor dauerhaft und sicher zugeordnet. Langfristig erhoffen sich die Autoren eine weitere Verbreitung von wissenschaftlichen Ideen und Entdeckungen, die bisher nur in sehr kleinen Kreisen veröffentlicht und diskutiert wurden.

Die Session „User Perceptions of Information Systems“ begann mit dem Vortrag von Tjaša Jug und Prof. Maja Žumer (Universität Ljubljana) „Content, Physical Appearance, Copy Condition - Tagging Customer Book Reviews“. Sie griffen die Problematik von unzureichend spezifizierten Online-Buchbeschreibungen und -bewertungen auf, die im Zeitalter von Amazon an Bedeutung gewinnen. Online-Buchhandlungen fassen alle Arten von Bewertungen unter dem Titel einer Arbeit zusammen. Dabei wird nicht auf die verschiedenen Abstraktionslevel eines $\mathrm{Bu}$ ches, die sogenannten Functional Requirements for Bibliographic Records (FRBR) eingegangen. In einer einzelnen Kritik wird so nicht deutlich, ob sich der Nutzer auf die Qualität des Inhalts bezieht oder beispielsweise mit dem Zustand des Einbandes eines gebrauchten Buches unzufrieden ist. Rückwirkend kann ein Käufer Bewertungen nach bestimmten Aspekten filtern (beispielsweise der $\mathrm{Zu}$ stand eines Werkes). Das ist ebenfalls aktuell nicht möglich. Um diesen Problemen beizukommen, entwickelten die Autoren die Methode des „Social Taggings“, bei denen 
einer Buchbewertung noch weitere Tags beigefügt werden können, um die Differenzierbarkeit und Organisation zu gewährleisten. Dazu wurden in drei Stufen Nutzern verschiedene modifizierte Buchbewertungen von Amazon präsentiert. Diese sollten sie zunächst selbst taggen. Anschließend wurden aus diesen freien Tags zehn Kategorien entwickelt und durch die Nutzer anschließend den Reviews wieder zugeordnet. Im letzten Schritt elvaluierte und verglich eine neue Benutzergruppe diese Zuordnungen. Die Studie zeigte, dass diese Art der Buchbewertung zwar die verschiedenen Attribute eines Buches erfasst, die Anwendbarkeit und Umsetzbarkeit in Online-Buchhandlungen jedoch noch unklar ist. Daher sind weitere Forschungen notwendig, um die Einsatzmöglichkeiten genauer zu identifizieren.

Danach stellten die beiden Autoren, Valerie Claessen und Adrian Schmidt, zusammen mit Dr. Tamara Heck (University of Southern Queensland) ihre Forschungsarbeit (mit dem Titel „Virtual Assistants: A Study on the Usability and User Perception of Customer Service System for ECommerce“ vor. Virtuelle Assistenten, auch Chatbots genannt, können Nutzer bei der Suche nach den richtigen Informationen unterstützen. Diese Programme verwenden Natural Language Processing, Lerntechniken und soziale Fähigkeiten, die eine messbare Nutzererfahrung möglich machen. Im E-Commerce werden diese virtuellen Assistenten als Unterstützung bei der Suche nach geeigneten Serviceinformationen oder Produkten angewendet. Diese Arbeit bewertet die Informationsdienstqualität von drei virtuellen Assistenten auf E-Commerce-Websites. Die zu analysierenden Aspekte decken die Servicequalität sowie die Nutzerwahrnehmung beim Umgang mit virtuellen Assistenten ab. Da zuvor keine Forschungsarbeiten dokumentiert wurden, die sich mit der Bewertung von virtuellen Assistenten befassen, musste ein Kriterienkatalog erstellt werden. Dieser basiert auf dem Information Service Evaluation Modell von Schumann und Stock. Damit konnte eine heuristische Analyse von Information Professionals durchgeführt werden. Im weiteren Verlauf der Forschungsarbeit wurde ein Nutzertest durchgeführt, mit dessen Daten die Usability-Erfahrung beim Umgang mit drei ausgewählten virtuellen Assistenten festhalten und mit der objektiven Analyse vergleichen konnten. Dies diente dazu, möglichen Ursachen für die Nutzerbewertung der einzelnen virtuellen Assistenten auf den Grund zu gehen. Erste Ergebnisse zeigen, dass die Technologie, die bei der Konstruktion eines virtuellen Assistenten verwendet wird, einen wesentlichen Einfluss auf die Nutzererfahrung hat. Ein virtueller Assistent, der mit einem anspruchsvollerem Natural Language Understanding und Processing bestückt war, wurde in der heuristischen Analyse deutlich höher als die anderen virtu- ellen Assistenten bewertet, die nur auf eingegebene Stichwörter reagierten. Die Interaktion mit diesem virtuellen Assistenten wurde von den Nutzern als intuitiver und daher angenehmer wahrgenommen. Allerdings zeigen die Ergebnisse, dass für eine wirklich zufriedenstellende UsabilityErfahrung die Nützlichkeit der Dienstleistungen verbessert werden muss.

Darauffolgend präsentierten Alexander von Janta Lipinski, Henrik Weber, Dr. Ralph Kölle und Prof. Thomas Mandl ihre Arbeit „Gamification Elements and Their Perception by Different Gamer Types“. Hintergrund ist das verstärkte Einsetzen von Gamification in Informationssystemen. Viele Entwickler bemühen sich, Gamification so effizient wie möglich in ein System einzubauen, um die Nutzer zu motivieren und die Produktivität bei der Arbeit zu steigern. Diese Forschungsarbeit dokumentiert einen Nutzertest, in dem die Nutzerwahrnehmung von drei Gamification-Elementen, u.a. verschiedene Belohnungssysteme, im Kontext eines Projektmanagements erfasst und auswertet wurden. Anhand der Projektmanagementsoftware „RedCritter Tracker“ wurde die Testumgebung erzeugt. Diese Software bietet ähnliche Funktionen wie herkömmliche Social-Media-Plattformen, sodass die eigenen Erfolge von anderen Nutzern eingesehen werden können. Nachdem die Nutzer einer Spielerkategorie zugeordnet werden konnten, wurde ihnen ein Testszenario zugewiesen. Durch das Vollenden von Aufgaben wurden ihnen auf verschiedene Weise Belohnungen zugeteilt. Belohnungspunkte wurden als motivierend wahrgenommen, wobei eine Bestenliste als kritisch betrachtet wurde. „Badges“ (Abzeichen) wurden als ineffektiv eingestuft. Es wird vermutet, dass die Einschätzung dieser verschiedenen Belohnungsprägungen auf dem Spielertyp zurückzuführen ist. Der sogenannte „Killer“ (Mörder) steht aufgrund seines Strebens nach Erfolg, Belohnungssystemen generell positiv gegenüber. Noch stärker empfindet der „Achiever“ (Leistungsbringer) Belohnungssysteme als positiv.

Der Dienstag endete mit der Verleihung des GerhardLustig-Preises für die beste studentische Masterarbeit im Bereich Informationswissenschaft. Nominiert waren: Fabian Muster, (HTW Chur, Schweizerisches Institut für Informationswissenschaft) mit „Data Strategy Model - a Reference Model to Develop Data Strategies”, Roland List (Universität Graz, Institut für Informationswissenschaft und Wirtschaftsinformatik) mit „Relationship Between Downloads and Citations", Alexandra Linhart (HAW Hamburg, Department Information) mit „Google Now and User Data”, Helen Brinken und Helena Kock (Universität Hildesheim, Institut für Informationswissenschaft \& Sprachtechnologie) mit „Exploring Intergenerational Knowledge Sharing in Organizations - Potentials and Limitations of 


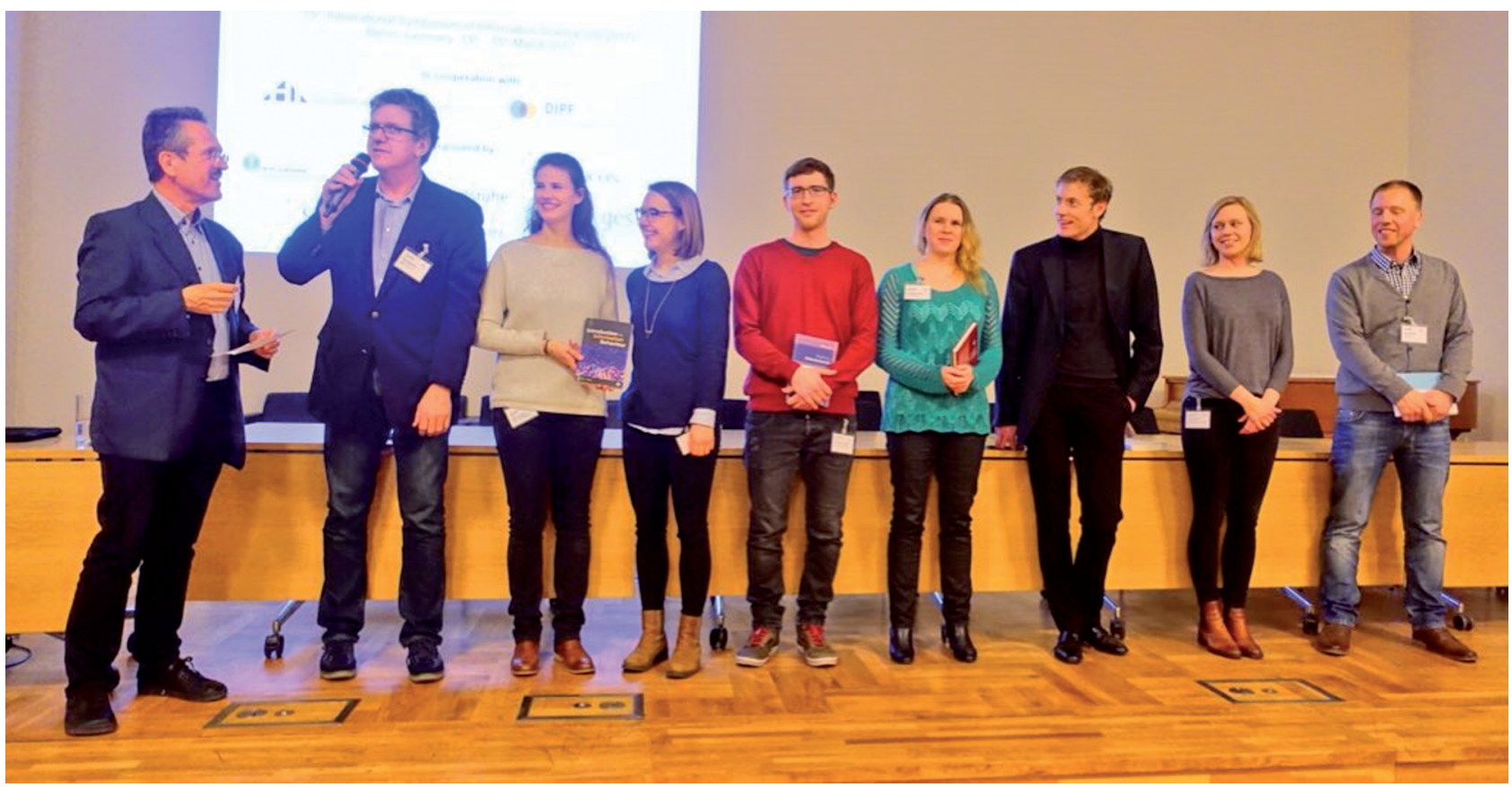

Abbildung 1: Die Nominierten des Gerhard-Lustig-Preis zusammen mit Prof. Dr. Achim Oswald und Prof. Dr. Marc Rittberger. (Bild: Valerie Claessen und Adrian Schmidt).

Information and Communication Technology (ICT)", Sandra Balck (Fachhochschule Potsdam, Fachbereich Informationswissenschaften) mit ,The X(Disciplinarity) of Information Science" und Robert Jackermeier (Universität Regensburg, Medieninformatik) mit „Interactive Pedestrian Indoor Localization”. Alle hatten ihre Arbeiten am selben Tag bereits vor einer Jury präsentiert. Auf der Abendveranstaltung wurden nun die Gewinner vorgestellt. Die Studentinnen der Universität Hildesheim, Helene Brinken und Helena Kock überzeugten mit ihrer gemeinsamen Arbeit und belegten den ersten Platz.

Deskriptoren: Informationswissenschaft, Konferenz, Informationssystem

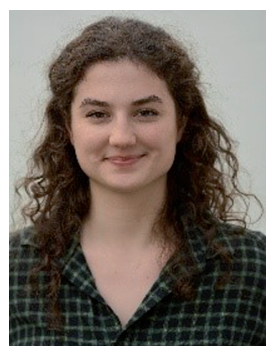

\section{Valerie Claessen}

Heinrich-Heine Universität Düsseldorf

Institut für Sprache und Information

Abteilung Informationswissenschaft

Universitätsstr. 1

40225 Düsseldorf

valerie.claessen@uni-duesseldorf.de

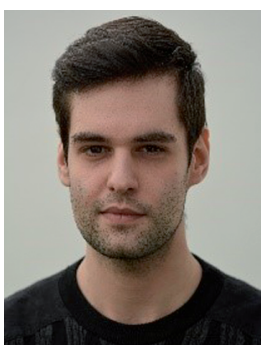

Adrian Schmidt

Heinrich-Heine Universität Düsseldorf Institut für Sprache und Information Abteilung Informationswissenschaft Universitätsstr. 1

40225 Düsseldorf

adrian.schmidt@uni-duesseldorf.de

Adrian Schmidt (geboren 1990) ist Masterstudent der Informationswissenschaft und Sprachtechnologie der Heinrich-Heine-Universität Düsseldorf. In seiner Bachelorarbeit behandelte er das Thema InGame Advertising. Studienbegleitend arbeitet er bei Kauz Lingustic Technologies.
Valerie Claessen (geboren 1993) ist Masterstudentin der Informationswissenschaft und Sprachtechnologie der Heinrich-Heine-Universität Düsseldorf. Studienbegleitend arbeitet sie bei Kauz Linguistic Technologies und schrieb ihre Bachelorarbeit über virtuelle Assistenten. 